\title{
ON THE GROWTH OF SOLUTIONS OF LINEAR DIFFERENTIAL EQUATIONS
}

\author{
J. ERNEST WILKINS, JR.
}

1. Introduction. In a recent paper by Boas, Boas and Levinson [1 $]^{1}$ two sets of sufficient conditions were given for the existence of $\lim _{x=\infty} y^{\prime}(x)$ when $y(x)$ satisfies the differential equation

$$
y^{\prime \prime}+A(x) y=B(x) \text {. }
$$

We propose in this paper to use their methods and to generalize their results to the $n$th order linear differential equation

$$
y^{(n)}+\sum_{i=1}^{n} A_{i}(x) y^{(n-i)}=B(x)
$$

and to obtain sufficient conditions for

$$
\lim _{x=\infty} y^{(n-1)}(x)
$$

to exist. In case $n=2, A_{1}(x)=0$ and $A_{2}(x)=A(x)$, these conditions reduce to those in [1].

2. Statements of the theorems. In $\$ 4$ we shall prove the following theorem.

Theorem I. If $A_{i}(x)(i=1, \cdots, n)$ and $B(x)$ are continuous on $0 \leqq x<\infty$, and if the integrals

$$
\begin{aligned}
& \int_{0}^{\infty} x^{i-1}\left|A_{i}(x)\right| d x \quad(i=1, \cdots, n), \\
& \int_{0}^{\infty} B(x) d x
\end{aligned}
$$

exist, then the limit $(1: 3)$ exists for any solution $y(x)$ of $(1: 2)$.

We now write each function $A_{i}(x)$ as the difference of two nonnegative functions, $A_{i}(x)=A_{i}^{\prime}(x)-A_{i}^{\prime \prime}(x)$, where $A_{i}^{\prime}=\left(\left|A_{i}\right|+A_{i}\right) / 2$, $A_{i}^{\prime \prime}=\left(\left|A_{i}\right|-A_{i}\right) / 2$. Then in $\$ 5, \cdots, \S 8$ we shall prove the following theorem.

TheOREM II. If $A_{i}(x)(i=1, \cdots, n)$ and $B(x)$ are continuous on $0 \leqq x<\infty$, if the integrals

Received by the editors October 30, 1943.

1 Numbers in brackets refer to the Bibliography at the end of the paper. 


$$
\begin{aligned}
& \int_{0}^{\infty} x^{i-1} A_{i}^{\prime \prime}(x) d x \quad(i=1, \cdots, n) \\
& \int_{0}^{\infty} B(x) d x
\end{aligned}
$$

exist, and if we have

$$
\limsup _{x=\infty} x^{i-k-1} \int_{x}^{\infty} t^{k} A_{i}^{\prime}(t) d t<2(i-k-1) ! k ! / n(n-1)
$$

whenever $i=2, k=0$, or $i=2 j-1,2 j, k=i-j, \cdots, i-2(j=2, \cdots$, $[(n+1) / 2])$, where we agree that $A_{n+1}^{\prime}(t)=0$ if $n$ is odd, then the limit $(1: 3)$ exists for any solution $y(x)$ of $(1: 2)$. If in addition we have

$$
\int_{0}^{\infty} \sum_{i=1}^{n} x^{i-1} A_{i}^{\prime}(x) d x=\infty \text {, }
$$

then $\lim _{x=\infty} y^{(n-1)}(x)=0$.

3. Some auxiliary lemmas. In this section we state four lemmas needed in proving the main theorems. The first of these is found in [1].

Lemma 1. If $f(x)$ is continuous on $0 \leqq x<\infty$, if $M(x)$ denotes the maximum of $|f(t)|$ on $0 \leqq t \leqq x$, and if, for some positive numbers $\alpha$ and $x_{0},|f(x)| \leqq \alpha+M(x) / 2\left(x \geqq x_{0}\right)$, then $f(x)$ is bounded on $0 \leqq x<\infty$.

LEMMA 2. If (2:5) holds under the restrictions on $i$ and $k$ stated in Theorem II, then (2:5) also holds for $i=2, \cdots, n, k=0, \cdots, i-2$.

This is manifestly true for $i=2$. If $i>2$ and if $i$ is odd, then $i=2 j-1, j \geqq 2$ and $(2: 5)$ holds if $k \geqq i-j=j-1$. Suppose now that $0 \leqq k<j-1$. Then $i-k-1=2 j-2-k>j-1$, and

$$
\begin{aligned}
x^{i-k-1} \int_{x}^{\infty} t^{k} A_{i}^{\prime}(t) d t & \leqq x^{j-1} \int_{x}^{\infty} t^{j-1} A_{i}^{\prime}(t) d t, \\
\limsup _{x=\infty} x^{i-k-1} \int_{x}^{\infty} t^{k} A_{i}^{\prime}(t) d t & <2(j-1) !^{2} / n(n-1) \\
& <2(2 j-2-k) ! k ! / n(n-1) .
\end{aligned}
$$

The reasoning when $i$ is even is quite similar.

LEMMA 3. If $f(x)$ is continuous and bounded on $0 \leqq x<\infty$, and if the functions $A_{i}(x)$ satisfy the hypotheses of Theorem $I$, then all integrals of the form 


$$
\int_{0}^{\infty} f(x) x^{p} A_{i}(x) d x, \quad \int_{0}^{\infty} x^{p}\left|A_{i}(x)\right| d x \quad(p=0, \cdots, i-1)
$$

exist. Under the hypotheses of Theorem II, the same conclusion is valid provided that $i=2, \cdots, n, p=0, \cdots, i-2$, or that $A_{i}(x)$ be replaced by $A_{i}^{\prime \prime}(x)$.

The proof of the first sentence of the lemma is immediate, and the second sentence follows similarly as soon as we refer to the preceding lemma.

Lemma 4. If $y(x)$ is of class $C^{(m+q)}$ on $0 \leqq x<\infty$, where $m$ and $q$ are non-negative integers, then

$$
q ! \limsup _{x=\infty} x^{-q}\left|y^{(m)}(x)\right| \leqq \limsup _{x=\infty}\left|y^{(m+q)}(x)\right| .
$$

To prove Lemma 4 we use Taylor's Theorem in the form

$$
\begin{aligned}
y^{(m)}(x)= & \sum_{k=0}^{q-1}\left(x-x_{0}\right)^{k} y^{(m+k)}\left(x_{0}\right) / k ! \\
& +\int_{x_{0}}^{x} \int_{x_{0}}^{t_{q-1}} \cdots \int_{x_{0}}^{t_{1}} y^{(m+q)}\left(t_{0}\right) d t_{0} d t_{1} \cdots d t_{q-1} .
\end{aligned}
$$

Let $M=\lim \sup _{x=\infty}\left|y^{(m+q)}(x)\right|$ and pick $e>0$. Then take $x_{0}$ so large that

$$
\left|y^{(m+q)}(x)\right|<M+e \quad\left(x \geqq x_{0}\right) .
$$

It follows from (3:2) that if $x \geqq x_{0}$

$$
\begin{gathered}
\left|y^{(m)}(x)\right| \leqq \sum_{k=0}^{q-1} x^{k}\left|y^{(m+k)}\left(x_{0}\right)\right| / k !+(M+e) x^{q} / q !, \\
q ! \limsup _{x=\infty} x^{-q}\left|y^{(m)}(x)\right| \leqq M+e .
\end{gathered}
$$

Since $e$ is arbitrary, the statement of the lemma follows at once.

4. Proof of Theorem I. By virtue of (2:1) we can pick $x_{0}$ such that

$$
\int_{x_{0}}^{\infty} \sum_{i=1}^{n} x^{i-1}\left|A_{i}(x)\right| /(i-1) ! d x<1 / 2
$$

If in $(1: 2)$ we substitute for $y^{(n-i)}(i=2, \cdots, n)$ the values obtained from (3:2) by replacing $m$ by $n-i$ and $q$ by $i-1$, and solve the resulting equation for $y^{(n)}$, we get an equation which upon integration between the limits $x_{0}$ and $x$ gives 


$$
\begin{aligned}
y^{(n-1)}(x)= & y^{(n-1)}\left(x_{0}\right)-\sum_{i=2}^{n} \sum_{k=0}^{i-2} \int_{x_{0}}^{x} A_{i}(t) \frac{\left(t-x_{0}\right)^{k}}{k !} y^{(n-i+k)}\left(x_{0}\right) d t \\
& -\sum_{i=1}^{n} \int_{x_{0}}^{x} \int_{x_{0}}^{t_{i-1}} \cdots \int_{x_{0}}^{t_{1}} A_{i}\left(t_{i-1}\right) y^{(n-1)}\left(t_{0}\right) d t_{0} \cdots d t_{i-1} \\
& +\int_{x_{0}}^{x} B(t) d t .
\end{aligned}
$$

Define the quantities $B$ and $\alpha$ and the function $M(x)$ by the equations

$$
\begin{aligned}
B= & \max _{x_{0} \leqq x \leqq \infty}\left|\int_{x_{0}}^{x} B(t) d t\right|, \\
\alpha= & \left|y^{(n-1)}\left(x_{0}\right)\right| \\
& +\sum_{i=2}^{n} \int_{x_{0}}^{\infty}\left|A_{i}(t)\right| \sum_{k=0}^{i-2} \frac{t^{k}}{k !}\left|y^{(n-i+k)}\left(x_{0}\right)\right| d t+B, \\
M(x)= & \max _{0 \leqq t \leqq x}\left|y^{(n-1)}(t)\right| .
\end{aligned}
$$

$B$ exists by virtue of $(2: 2)$ and $\alpha$ exists by virtue of Lemma 3 . From $(4: 2)$ and $(4: 1)$ we now get

$$
\left|y^{(n-1)}(x)\right| \leqq \alpha+M(x) / 2 \quad\left(x \geqq x_{0}\right) .
$$

It follows from Lemma 1 that $y^{(n-1)}(x)$ is bounded on $0 \leqq x<\infty$. In this event we use Lemma 3 to see that the integrals involving $A_{i}(t)$ on the right side of $(4: 2)$ approach limits as $x \rightarrow \infty$. By $(2: 2)$ the integral of $B(x)$ approaches a limit. Therefore, $y^{(n-1)}(x)$ has a limit, proving Theorem I.

5. Proof of Theorem II when $y^{(n-1)}(x)$ does not change sign for large values of $x$. Then we may assume without loss of generality that $x_{0}$ is so large that $y^{(n-1)}(x) \geqq 0$ for $x \geqq x_{0}$ and that

$$
\int_{x_{0}}^{\infty} \sum_{i=1}^{n} x^{i-1} A_{i}^{\prime \prime}(x) /(i-1) ! d x<1 / 2 .
$$

Since $y^{(n-1)}(t) \geqq 0$ on $t \geqq x_{0}$ and $-A_{i}(t)=A_{i}^{\prime \prime}(t)-A_{i}^{\prime}(t) \leqq A_{i}^{\prime \prime}(t)$, we then have from $(4: 2)$ and $(5: 1)$ that (4:4) holds, the integrals in $\alpha$ existing by virtue of the second part of Lemma 3 . It follows from Lemma 1 that $y^{(n-1)}(x)$ is bounded. Set $A_{i}=A_{i}^{\prime}-A_{i}^{\prime \prime}$ in (4:2). Since $y^{(n-1)}(x)$ is bounded we see from Lemma 2 that all of the terms in (4:2) on the right side approach limits as $x \rightarrow \infty$ with the possible exception of 


$$
-\sum_{i=1}^{n} \int_{x_{0}}^{x} \int_{x_{0}}^{t_{i-1}} \cdots \int_{x_{0}}^{t_{1}} A_{i}^{\prime}\left(t_{i-1}\right) y^{(n-1)}\left(t_{0}\right) d t_{0} \cdots d t_{i-1} .
$$

Since $A_{i}^{\prime} \geqq 0, y^{(n-1)}\left(t_{0}\right) \geqq 0$ for $t_{0} \geqq x_{0}$, this term is a nonincreasing function of $x$ which is bounded below since all the other terms in $(4: 2)$ are bounded. Hence it also approaches a limit. Therefore, the limit $(1: 3)$ exists.

6. Proof of Theorem II when $y^{(n-1)}(x)$ changes sign infinitely many times. Suppose first that $y^{(n-1)}(x)$ is bounded but that the limit $(1: 3)$ does not exist. Then we may assume without loss of generality that

$$
\lim \sup \left|y^{(n-1)}(x)\right|=\lim \sup y^{(n-1)}(x)=M>0 .
$$

Let $x_{m}$ be a monotone sequence of points such that $x_{m} \rightarrow \infty, y^{(n-1)}\left(x_{m}\right)$ $>0, y^{(n-1)}\left(x_{m}\right) \rightarrow M$. Let $a_{m}$ be the first point to the left of $x_{m}$ such that $y^{(n-1)}\left(a_{m}\right)=0$. We can suppose that $a_{1}$ is so large that for some $c<1$ and for $i=2, \cdots, n, k=0, \cdots, i-2$ we have

$$
x^{i-1-k} \int_{x}^{\infty} t^{k} A_{i}^{\prime}(t) d t<2 c(i-1-k) ! k ! / n(n-1) \quad\left(x \geqq a_{1}\right) .
$$

By (4:2) with $x_{0}$ replaced by $a_{m}$ and $x$ replaced by $x_{m}$ we have, if we observe that $y^{(n-1)}(t) \geqq 0$ on $a_{m} \leqq t \leqq x_{m}$,

$$
\begin{aligned}
y^{(n-1)}\left(x_{m}\right) \leqq & \sum_{i=1}^{n} \int_{a_{m}}^{x_{m}} \int_{a_{m}}^{t_{i-1}} \cdots \int_{a_{m}}^{t_{1}} A_{i^{\prime \prime}}\left(t_{i-1}\right) y^{(n-1)}\left(t_{0}\right) d t_{0} \cdots d t_{i-1} \\
& +\sum_{i=2}^{n} \sum_{k=0}^{i-2} \int_{a_{m}}^{x_{m}} \frac{t^{k}}{k !} A_{i^{\prime \prime}}(t)\left|y^{(n-i+k)}\left(a_{m}\right)\right| d t \\
& +\sum_{i=2}^{n} \sum_{k=0}^{i-2} \int_{a_{m}}^{x_{m}} \frac{t^{k}}{k !} A_{i}^{\prime \prime}(t)\left|y^{(n-i+k)}\left(a_{m}\right)\right| d t \\
& +\left|\int_{a_{m}}^{x_{m}} B(t) d t\right| .
\end{aligned}
$$

Since $y^{(n-1)}(t)$ is bounded, we see from Lemma 3 that the first sum on the right of $(6: 2)$ approaches zero as $m \rightarrow \infty$. By virtue of $(2: 4)$ so does the last term in $(6: 2)$. If we use Lemma 4 we discover that the upper limit of the third sum in $(6: 2)$ can not exceed

$$
\begin{aligned}
\underset{m=\infty}{\limsup } \sum_{i=2}^{n} \sum_{k=0}^{i-2} \frac{\left|y^{(n-i+k)}\left(a_{m}\right)\right|}{k ! a_{m}^{i-1-k}} \int_{a_{m}}^{x_{m}} t^{i-1} A_{i}^{\prime \prime}(t) d t \\
\leqq \limsup _{x=\infty} \sum_{i=2}^{n} \sum_{k=0}^{i-2} \frac{\left|y^{(n-1)}(x)\right|}{k !(i-1-k) !} \int_{x}^{\infty} t^{i-1} A_{i}^{\prime \prime}(t) d t=0 .
\end{aligned}
$$


Finally we use (6:1) and Lemma 4 to see that the upper limit of the second sum in $(6: 2)$ cannot exceed

$$
\begin{aligned}
\underset{m=\infty}{\limsup } \sum_{i=2}^{n} \sum_{k=0}^{i-2} & \int_{a_{m}}^{\infty} \frac{t^{k}}{k !} A_{m}^{\prime}(t)\left|y^{(n-i+k)}\left(a_{m}\right)\right| d t \\
& \leqq \limsup _{x=\infty} \sum_{i=2}^{n} \sum_{k=0}^{i-2} \frac{2 c(i-1-k) !\left|y^{(n-i+k)}(x)\right|}{n(n-1) x^{i-1-k}} \\
& \leqq \limsup _{x=\infty} \sum_{i=2}^{n} \sum_{k=0}^{i-2} 2 c\left|y^{(n-1)}(x)\right| / n(n-1)=c M<M
\end{aligned}
$$

Referring to (6:2) we see that we have reached a contradiction of our choice of the points $x_{m}$.

7. Proof that $y^{(n-1)}(x)$ must be bounded. To complete the proof of the first part of Theorem II, it is sufficient to prove that $y^{(n-1)}(x)$ must be bounded under the hypotheses $(2: 3),(2: 4),(2: 5)$ and the assumption that $y^{(n-1)}(x)$ changes sign infinitely many times. Suppose on the contrary that $y^{(n-1)}$ is unbounded. Then we can pick a sequence $x_{m} \rightarrow \infty$ such that

$$
\left|y^{(n-1)}\left(x_{m}\right)\right| \geqq\left|y^{(n-1)}(x)\right| \quad\left(x \leqq x_{m}\right),
$$

$y^{(n-1)}\left(x_{m}\right)$ has the same sign, which we may suppose to be positive, and $y^{(n-1)}\left(x_{m}\right) \rightarrow \infty$. Let $a_{m}$ be defined for $x_{m}$ as in $\$ 6$, and suppose that $a_{1}$ is so large that (6:1) holds. Using (7:1) and Taylor's Theorem (3:2) with $m$ replaced by $n-i+k, q$ replaced by $i-1-k, x_{0}$ replaced by 0 , and $x$ replaced by $a_{m}$, we find that

$$
\left|y^{(n-i+k)}\left(a_{m}\right)\right| \leqq y^{(n-1)}\left(x_{m}\right) \frac{a_{m}^{i-k-1}}{(i-k-1) !}+\sum_{h=0}^{i-k-2}\left|y^{(n-i+k+n)}(0)\right| \frac{a_{m}^{h}}{h !} .
$$

It now follows from $(6: 2)$ and $(6: 1)$ that

$$
\begin{aligned}
y^{(n-1)}\left(x_{m}\right) \leqq & y^{(n-1)}\left(x_{m}\right)\left\{\sum_{i=1}^{n} \int_{a_{m}}^{\infty} \frac{t^{i-1} A_{i}^{\prime \prime}(t)}{(i-1) !} d t+c\right. \\
& \left.+\sum_{i=2}^{n} \sum_{k=0}^{i-2} \int_{a_{m}}^{\infty} \frac{t^{i-1} A_{i}^{\prime \prime}(t)}{k !(i-k-1) !} d t\right\}+\mid \int_{a_{m}}^{x_{m}} B(t) d t \\
& +\sum_{i=2}^{n} \sum_{k=0}^{i-2} \sum_{h=0}^{i-k-2} \frac{\left|y^{(n-i+k+h)}(0)\right|}{k ! h !} \int_{a_{m}}^{\infty} t^{k+h} A_{i}(t) d t .
\end{aligned}
$$

Since all of the integrals on the right of this last inequality approach zero as $m \rightarrow \infty$ and $0<c<1$, we reach a contradiction. 
8. Proof of the second part of Theorem II. Suppose on the contrary that $y^{(n-1)}(x)$ does not approach zero. Without loss of generality we may assume that

$$
\lim _{x=\infty} y^{(n-1)}(x)=2 a>0 .
$$

Then there exists an $x_{0}$ such that

$$
3 a>y^{(n-1)}(x)>a \quad\left(x \geqq x_{0}\right) .
$$

Now set $A_{i}=A_{i}^{\prime}-A_{i}^{\prime \prime}$ in $(4: 2)$ and let $x \rightarrow \infty$. Then all of the terms on the right approach limits with the possible exception of the term $(5: 2)$. Since $y^{(n-1)}(x)$ approaches a limit, so must $(5: 2)$. But by $(8: 1)$ we have

$$
\int_{x_{0}}^{t_{i-1}} \cdots \int_{x_{0}}^{t_{1}} y^{(n-1)}\left(t_{0}\right) d t_{0} \cdots d t_{i-2}>a\left(t_{i-1}-x_{0}\right)^{i-1} /(i-1) ! .
$$

Consequently, the term $(5: 2)$ is greater than

$$
a \int_{x_{0}}^{x} \sum_{i=1}^{n}\left(t-x_{0}\right)^{i-1} A_{i}^{\prime}(t) /(i-1) ! d t .
$$

By $(2: 6)$ and Lemma 3 this last integral becomes infinite as $x \rightarrow \infty$, so that $(5: 2)$ cannot approach a limit. This contradiction completes the proof of Theorem II.

Added in proof. Since the submission of this paper to the editors, it has come to the author's attention that Theorem I was proved by Otto Haupt, Über das asymptotische Nerhalten der Lösungen gewisser linearer gewöhnlicher Differentialgleichungen, Math. Zeit. vol. 48 (1942) pp. 282-292. Our proof, based on Lemma 1, seems distinctly simpler and certainly more elementary than that of Haupt. To the best of our present knowledge, Theorem II is new.

\section{BIBLIOGRAPHY}

1. Boas, Boas and Levinson, The growth of solutions of a differential equation, Duke Math. J. vol. 9 (1942) pp. 847-853.

TUskegee Institute 